Ann. Biol. anim. Bioch. Biophys., I974, 14 (2), 327-341.

\title{
MISE EN ÉVIDENCE ET CARACTÉRISATION PARTIELLE DE DIFFÉRENTES PEPTIDASES CHEZ SACCHAROMYCES LACTIS
}

\author{
M.-J. DESMAZEAUD et J.-J. DEVOYOD* \\ avec la collaboration technique de C. Auriault \\ Laboratoive de Biochimie microbienne, \\ Centre national de Recherches zootechniques, I. N. R. A., \\ 78350 Jouy en Josas \\ * Laboratoire de Recherches fromagères, I. N. R. A., \\ 15000 Aurillac
}

\section{RÉSUMÉ}

Après broyage des cellules aux ultrasons une activité de type carboxypeptidase (EC 3.4 .2 ) était mise en évidence à $\mathrm{pH}$ 7, o sur les substrats synthétiques Z-Gly-Leu, Z-Gly-Phe et Z-Glu-Tyr. Après élimination des acides nucléiques et précipitation au sulfate d'ammonium trois activités de type aminopeptidase (EC 3.4.I) étaient mises en évidence sur le substrat Leucine-para-nitroanilide (LNA) et isolées par chromatographies sur DEAE-cellulose et ECTEOLA-cellulose. Les aminopeptidases ( $\mathrm{AP}$ I, $\mathrm{AP} 2, \mathrm{AP} 3$ ) ont les caractères de métalloenzymes : l'EDTA et l'o-phénanthroline inhibent fortement les peptidases AP I et AP2, totalement l'enzyme AP 3. Cette dernière est aussi inactivée par la cystéine et le $\beta$-mercaptoéthanol. Les peptidases $\mathrm{AP}{ }_{1}, \mathrm{AP}_{2}, \operatorname{AP} 3$, ont respectivement comme $\mathrm{pH}$ optimum : 5, $\mathrm{O}, 7, \mathrm{I}$ et 8 , o et présentent une stabilité maximum de pH 7,o à 8,o. Leur température optimale d'hydrolyse de la LNA est de $4^{\circ}$ à $45^{\circ} \mathrm{C}$ (l'énergie apparente d'activation de la peptidase AP I est de Io ooo cal $/$ mole). L'enzyme AP 3 est stable à $50^{\circ} \mathrm{C}$; $50 \mathrm{p}$. roo d'activité sont détruits après une incubation à $70^{\circ} \mathrm{C}$ pendant $\mathrm{I} 5 \mathrm{mn}$. La peptidase AP I est stable jusqu'à $65^{\circ} \mathrm{C} ; 28 \mathrm{p}$. Ioo d'activité sont détruits après incubation à $70^{\circ} \mathrm{C}$ pendant Io mn. Ces peptidases présentent d'autre part une spécificité différente vis-à-vis de l'hydrolyse de nombreux peptides de synthèse.

\section{ABRÉVIATIONS}

Tris : Tris-(hydroxyméthyle)-aminométhane ;

DEAE : Diéthylaminoéthyle ;

ECTEOLA : Epichlorohydrine-triéthanolamine ;

LNA : Leucine-para-nitroanilide ;

Z : Benzyloxycarbonyle ; 
DO : Densité optique ;

EDTA : Acide éthylène diamine tétra-acétique (sel disodique);

p-CMB : Para-chloromercuribenzoate de sodium;

DFP : Diisopropyle fluorophosphate.

\section{INTRODUCTION}

Parmi les microorganismes qui constituent la flore naturelle du fromage de Roquefort les streptocoques lactiques, les Leuconostoc et les lactobacilles mésophiles ont des besoins nutritifs en peptides et en acides aminés, qui peuvent être assurés par des microorganismes protéolytiques (DEvoyod et DESMAZEAUD, I970 et I97I). Or, dans les fromages bleus, les levures, peu nombreuses en général dans le lait de fabrication se multiplient rapidement au cours des 24 premières heures qui suivent l'emprésurage. A ce stade on rencontre des levures fermentant le lactose dont l'espèce Saccharomyces lactis (Devoyod et Sponem, I970). Puis au moment du salage ces levures se lysent. On peut se demander si la stimulation des bactéries lactiques constatée à ce stade de la fabrication n'est pas due aux peptides et acides aminés libérés lors de cette lyse cellulaire. C'est la raison qui nous a conduit à entreprendre l'étude du système peptidasique intracellulaire de $S$. lactis.

A notre connaissance aucune détermination précise des activités protéolytiques ou peptidasiques n'a été effectuée chez S. lactis. Par contre chez Saccharomyces cerevisiae plusieurs types de protéinases (FELIX et BrouILLET, I966 ; HATA, HAYAshi et Dor, I967; LENNEY et DALBEC, I967) ou de carboxypeptidases (HAyashi, Aibara et HATA, I970 ; FÉLIX et Brouil,LET, I966) ont été isolés et caractérisés. De même, chez S. cerevisiae var. ellipsoideus (CoRDONNIER, I966) l'activité amino-carboxypeptidasique (dipeptidase) a été étudiée de façon approfondie. De plus, quatre enzymes protéolytiques ont été caractérisées chez Saccharomyces carlsbergensis (MaDdox et HoUGH, I970). Enfin, une protéase acide exocellulaire a été purifiée et ses propriétés déterminées chez Rhodotorula glutinis (Kamada, OdA et Murao, I972).

\section{TECHNIQUES EXPÉRIMENTALES ÉT MATÉRIEL UTILISÉ:}

\section{Organisme}

Saccharomyces lactis souche SL 3 IO. Les caractéristiques biochimiques de cette souche ont été décrites antérieurement (DEvoyon et SPONEM, I970).

\section{Extrait cellulaive}

Les $330 \mathrm{~g}$ de cellules provenaient de I60 litres d'une culture sur un milieu à l'extrait de malt (Malt Extract Broth Difco (no I I 3 ) : 30 g ; yeast extract Difco : 1, 6 g ; q. s. I ooo ml d'eau distillée) ajusté à $\mathrm{pH}_{4,50}$ avec de l'acide lactique concentré. Après une incubation de 40 heures à $22^{\circ} \mathrm{C}$ les cellules étaient récoltées par centrifugation à $\mathbf{I} 7000 \mathrm{~g}$ (centrifugeuse Sharples réfrigérée). La croissance étant contrôlée par mesure de la DO des cultures à $650 \mathrm{~nm}$, ce temps permet d'atteindre la phase stationnaire pour laquelle la plus grande activité peptidasique était détectée (essais préliminaires non reportés dans ce mémoire). Les cellules étaient lavées deux fois par du tampon 
Tris-maléate de sodium o,005 M à $\mathrm{pH}$ 7, Io et remises en suspension dans ce même tampon, puis soumises à un traitement aux ultrasons dans un appareil Siduse US-77-7 à une fréquence de $23 \mathrm{Khz}$ pendant $\mathrm{I} 5 \mathrm{mn}$; la température pendant le traitement était maintenue à $4^{\circ} \mathrm{C}$. La suspension cellulaire était ensuite centrifugée à $27000 \mathrm{~g}$ pendant $\mathrm{I}$ heure à $4^{\circ} \mathrm{C}$ et le surnageant conservé (surnageant $\mathrm{S}$ r).

\section{Procédé d'isolement et de purification partielle des aminopeptidases}

\section{1re étape: Élimination des acides nucléiques.}

Les acides nucléiques étaient hydrolysés en ajoutant à I I $5^{\circ} \mathrm{ml}$ du surnageant $\mathrm{S}$ I, $3^{8} \mathrm{mg}$ de ribonucléase (Sigma), o,8 $\mathrm{mg}$ de désoxyribonucléase (Sigma) et $2 \mathrm{~g}$ de chlorure de magnésium. Après incubation pendant 2 heures à $30^{\circ} \mathrm{C}$ avec agitation lente, $12,5 \mathrm{~g}$ de sulfate de manganèse étaient ajoutés. Après I heure à $4^{\circ} \mathrm{C}$ le précipité était éliminé par centrifugation à ro ooo g pendant Io minutes à $4^{\circ} \mathrm{C}$ et le surnageant $\left(\mathrm{S}_{2}\right)$ recueilli.

\section{2 e étape: Précipitations au sulfate d'ammonium.}

L'opération était effectuée en deux étapes. Une première précipitation était obtenue après addition de $29 \mathrm{I} \mathrm{g}$ de sulfate d'ammonium à I $200 \mathrm{ml}$ de surnageant $\mathrm{S} 2$ (soit $40 \mathrm{p}$. Ioo de saturation en $\left.\left(\mathrm{NH}_{4}\right)_{2} \mathrm{SO}_{4}\right)$; après 90 minutes à $4^{\circ} \mathrm{C}$ sans agitation, le précipité était éliminé par centrifugation à 10 ooo g pendant 30 minutes à $4^{\circ} \mathrm{C}$; seul le surnageant S 3 contenait une activité peptidasique. Une deuxième précipitation était effectuéc sur I $360 \mathrm{ml}$ de ce dernier surnageant par addition de $387 \mathrm{~g}$ de sulfate d'ammonium (soit $80 \mathrm{p}$. Ioo de saturation en sulfate d'ammonium) après $\mathrm{I}_{5}$ heures à $4^{\circ} \mathrm{C}$ sans agitation, le précipité, qui contient l'activité peptidasique, était recueilli par centrifugation à $\mathrm{I} o$ ooo $\mathrm{g}$ pendant 30 minutes à $4^{\circ} \mathrm{C}$ et redissous (extrait $\mathrm{E} \mathrm{I}$ ) dans environ $\mathrm{I} 30 \mathrm{ml}$ de tampon Tris-maléate de sodium $0,005 \mathrm{M}$ à $\mathrm{pH} 7, \mathrm{IO}$.

\section{3e étape: Chromatographie sur gel de Sepharose 6 B.}

Le gel de Sepharose $6 \mathrm{~B}$ (Sephadex) était abondamment lavé sur un filtre en verre fritté $\mathrm{G}_{2}$ par le tampon Tris-maléate de sodium o,o05 $\mathrm{M}$ à $\mathrm{pH} 7$, Io puis versé dans une colonne de chromatographie. Soixante-cinq millilitres d'extrait peptidasique $\mathrm{E}_{1}$ préalablement dialysés pendant I6 heures à $4^{\circ} \mathrm{C}$ contre le tampon Tris-maléate étaient ensuite déposés au sommet de cette colonne. Les fractions étaient recueillies au moyen d'un collecteur GME et l'on enregistrait en continu la densité optique à $280 \mathrm{~nm}$ à l'aide d'un spectrophotomètre enregistreur Seive. On réunissait celles présentant l'activité peptidasique la plus forte (extrait F) et l'on concentrait les protéines dans une cellule Diaflo (Amicon) sur une membrane UM ro Diaflo.

\section{4 étape: Chromatographie sur DEAE-cellulose.}

Vingt millilitres d'extrait $F$ étaient déposés au sommet d'une colonne de DEAE-cellulose DE-23 (Whatman) préalablement équilibrée en tampon Tris-maléate de sodium o,005 $\mathrm{M}$ à $\mathrm{pH}$ 7, Io. Après lavage de la colonne par $226 \mathrm{ml} \mathrm{du}$ tampon d'équilibrage l'élution était réalisée par application d'un gradient linéaire de concentration en chlorure de sodium en tampon Tris-maléate de sodium o,o05 $\mathrm{M}$ à $\mathrm{pH} 7$, ro. Les fractions étaient recueillies et dosées comme décrit ci-dessus puis réunies en quatre extraits: $\mathrm{F}_{\mathrm{r}}, \mathrm{F}_{2}, \mathrm{~F}_{3}$ et $\mathrm{F}_{4}$.

$$
5^{\mathrm{e}} \text { étape: }
$$

\section{a) Chromatographie sur ECTEOLA-cellulose.}

La fraction $\mathrm{F} 3$ après concentration sur membrane Diaflo UM ro et dialyse pendant 16 heures à $4^{\circ} \mathrm{C}$ contre le tampon Tris-maléate était déposée au sommet d'une colonne d'ECTEOLA-cellulose E 9 I 26 (Sigma) préparée en tampon Tris-maléate de sodium 0,005 M à pH 7, Io. Après lavage de la colonne par $194 \mathrm{ml}$ du tampon d'équilibrage, l'élution était réalisée par application d'un gradient linéaire de concentration en chlorure de sodium dans ce même tampon. Les fractions étaient recueillies et dosées comme précédemment puis réunies en trois extraits $\mathrm{F} 3 a, \mathrm{~F} 3 b$ et F $3 c$

\section{b) Chromatographie sur Bio-Gel P-100.}

La fraction $\mathrm{F}_{4}$ était chromatographiée sur une colonne $(75 \times 3.5 \mathrm{~cm})$ de Bio-Gel P-Ioo (Bio-Rad Laboratories) préalablement équilibrée dans le tampon Tris-maléate de sodium o,oo5 M à $\mathrm{pH}$ 7, ro. Les fractions étaient recueillies et dosées comme décrit ci-dessus et divisées en deux extraits peptidasiques actifs sur les peptides de caséine : $\mathrm{F}_{4} a$ et $\mathrm{F}_{4} b$. 
6e étape: Chromatographie sur gel de Sephadex G-100.

Les différentes fractions actives ainsi isolées étaient finalement chromatographiées sur une colonne $(98 \mathrm{~cm} \times 2$ ) de Sephadex G-Ioo équilibrée en tampon Tris-maléate de sodium $0,005 \mathbf{M}$ à $\mathrm{pH}$ 7, ro. La colonne était étalonnée avec le Blue-dextran 2000 (Sephadex), la sérum-albumine bovine (Sigma) et la ribonucléase (Calbiochem).

\section{Dosage des activités peptidasiques}

Origine des substrats.

Tous les produits utilisés sont de forme $\mathrm{L}$.

- Amides d'acides aminés : Cyclo; leucine-para-nitro-anilide (LNA) : Sigma ;

- Dipeptides non substitués : Gly-Gly : NBC; Gly-Phe : Mann; tous les autres : Cyclo;

-Dipeptides monosubstitués : Z-Gly-Phe : Fluka; Z-Gly-Ala ; Z-Gly-Leu et Z-Glu-Tyr : Cyclo.

- Dipeptides disubstitués : Z-Gly-Tyr-NH $\mathrm{N}_{2}, \mathrm{Z}-\mathrm{His}-\mathrm{Phe}-\mathrm{NH}_{2}, \mathrm{Z}-\mathrm{Thr}-\mathrm{Phe}-\mathrm{NH}_{2}, \mathrm{Z}-\mathrm{Ala}-\mathrm{Phe}-$ $\mathrm{NH}_{2}$ : Cyclo ; Z-Gly-Leu-NH $\mathrm{N}_{2}$ : Mann.

- Tripeptides non substitués : Gly-Tyr-Gly, Gly-Trp-Gly : Cyclo et Gly-Gly-Gly-Gly : Mann.

-.- "Peptides de caséine à 0,02 p. IOO ": cette solution était obtenue par hydrolyse de la caséine entière par la protéase neutre de Micrococcus caseolyticus comme décrit précédemment (Desmazeaud et Hermier, 1972).

- Glucagon : Calbiochem.

\section{Dosage quantitatif des activités aminopeptidasiques}

- Substrat leucine-para-nitroanilide (LNA) (RoncARI et ZuBER, I969) : le mélange réactionnel avait la composition suivante : $0,1 \mathrm{ml}$ d'une solution de LNA en méthanol (à $6,4 \mathrm{mg}$ de LNA pour I $\mathrm{ml}$ de méthanol) ; $2,8 \mathrm{ml}$ de tampon Tris-maléate de sodium $0,005 \mathrm{M}$ à $\mathrm{pH} 7, \mathbf{1 0}$; $0, \mathrm{I} \mathrm{ml}$ de solution enzymatique. Après incubation à $37^{\circ} \mathrm{C}$ pendant différents intervalles de temps la réaction était arrêtée par addition de $0,5 \mathrm{ml}$ d'acide acétique à $30 \mathrm{p}$. Ioo (poids/volume). La variation de densité optique était mesurée à $4 \mathrm{I} 0 \mathrm{~nm}$. Bien que plus précise la méthode spectrophotométrique continue n'a pu être employée, le spectrophotomètre utilisé n'étant pas équipé de cuves thermostatées.

- Substrats dipeptides monosubstitués et " peptides de caésine " : le mélange réactionnel avait la composition suivante : $0,9 \mathrm{ml}$ de substrat à une concentration de $\mathrm{I} \mathrm{mM}$ pour les dipeptides monosubstitués ou à $0,02 \mathrm{p}$. I oo pour les "peptides de caséine " en tampon Tris-maléate de sodium $0,005 \mathrm{M}$ à $\mathrm{pH} 7, \mathrm{ro} ; 0, \mathrm{I} \mathrm{ml}$ de solution enzymatique. Après incubation à $37^{\circ} \mathrm{C}$ pendant différents intervalles de temps, la réaction était arrêtée en portant le mélange réactionnel au bain-marie bouillant pendant $\mathrm{I} 5 \mathrm{mn}$ après addition de $\mathrm{I} \mathrm{ml}$ du réactif à la ninhydrine (Moore et S'TEIN, I954). La variation de densité optique était mesurée à $570 \mathrm{~nm}$ après dilution dans 1'alcool à 50 p. IoO.

\section{Dosage qualitatif des activités amino- ou carboxypeptidasiques}

Le mélange réactionnel avait la composition suivante : $0,1 \mathrm{ml}$ de substrat à une concentration de $\mathrm{I} \mathrm{mM}$ en tampon Tris-maléate de sodium $0,005 \mathrm{M}$ à $\mathrm{pH} 7,10 ; 0,1 \mathrm{ml}$ de solution de peptidase. Après incubation pendant différents intervalles de temps à $37^{\circ} \mathrm{C}$ les mélanges réactionnels étaient déposés en spots sur une feuille de papier Whatman 3 MM et la détection des peptides ou (et) des acides aminés éventuellement libérés par l'hydrolyse était effectuée après chromatographie descendante ou électrophorèse à haut voltage selon des techniques décrites ailleurs (DESMAZEAUD, 1972).

\section{RÉSULTATS}

\section{I. - Séparation des différentes aminopeptidases}

Après élimination des acides nucléiques par traitement aux nucléases, précipitations au sulfate d'ammonium et chromatographie sur Sépharose $6 \mathrm{~B}$, on ne détectait plus aucune activité carboxypeptidasique (tabl. I). L'utilisation d'un substrat 
TABLEAU I

Schéma d'isolement et de purification des différentes peptidases de $\mathrm{S}$. lactis

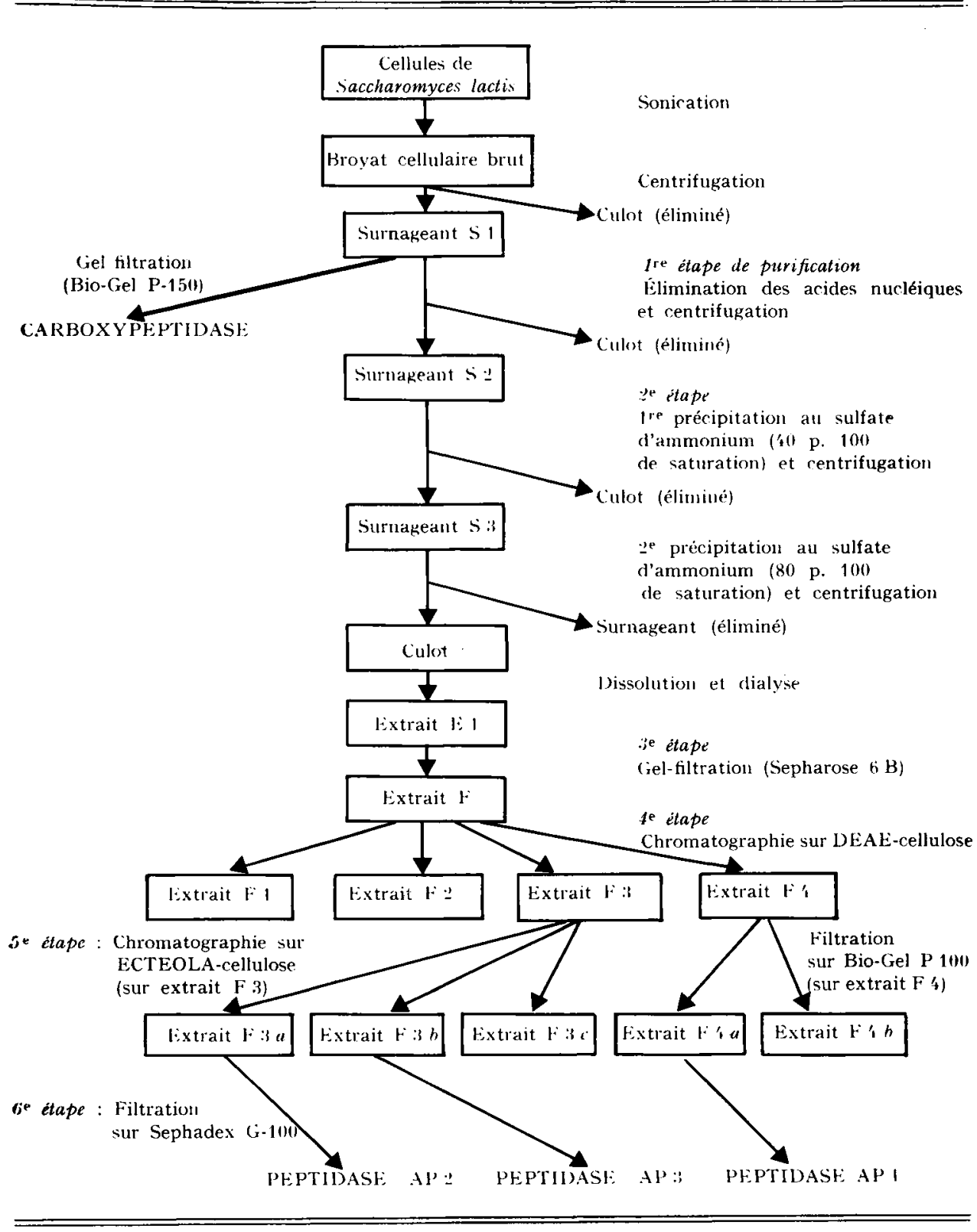


complexe (" peptides de caséine ") pour détecter l'activité après chromatographie sur Sépharose $6 \mathrm{~B}$, montre l'existence à ce stade de purification, de plusieurs peptidases (fig. I). Un gradient linéaire de concentration en chlorure de sodium permet l'élution distincte de quatre activités peptidasiques ( $\mathrm{F}$ I à $\mathrm{F}$ 4) sur DEAE-cellulose (fig. $2 \mathrm{~A}$ et tabl. I). Seules ont été retenues les fractions les plus actives $\mathrm{F} 3$ et F 4 . La fraction $\mathrm{F} 3$ était décomposée en trois extraits actifs par chromatographie sur ECTEOLA-cellulose (fig. 2 B et tabl. I) ; la plupart des protéines non actives étant

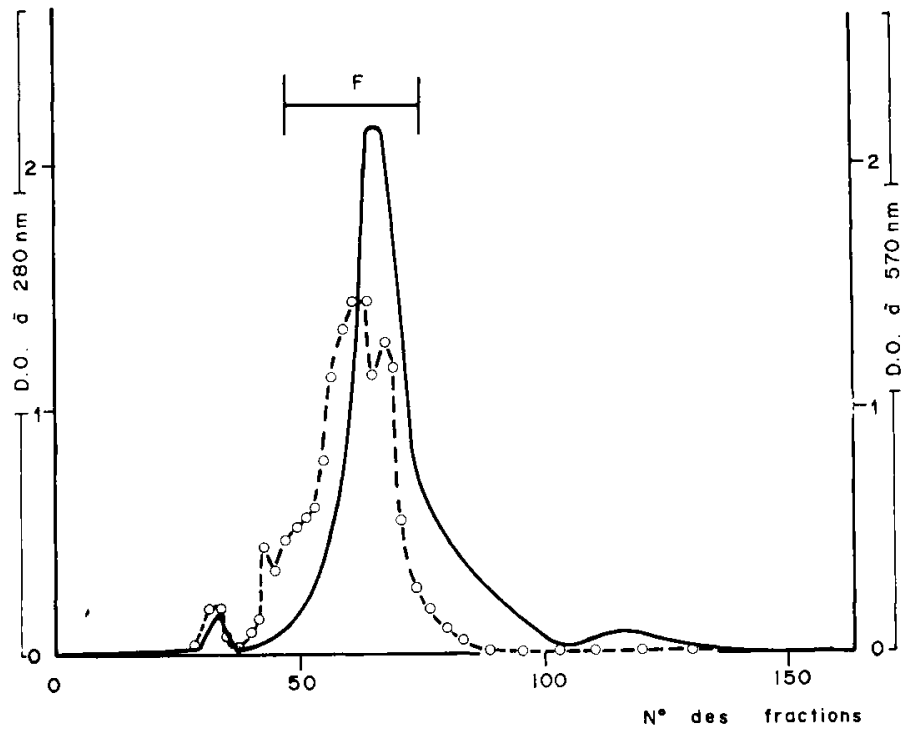

FIG. I. - Chromatographie sur Sepharose $6 B$ de l'extrait E 1 (après précipitation au sulfate d'ammonium)

Colonne roo $\times 4,5 \mathrm{~cm}$; débit $55 \mathrm{ml} / \mathrm{h}$; volume des fractions $15 \mathrm{ml}$; tampon Tris-maléate de sodium; $\mathrm{pH} 7,10$

$-\quad D O$ à $280 \mathrm{~nm}=$ élution des protéines;

o- - D DO à $570 \mathrm{~nm}=$ activité peptidasique (substrat : peptides de caséine).

éluées au début du gradient, ce fait explique la non-correspondancé entre les pics principaux de protéines et ceux obtenus après dosage sur la INNA; la fraction $\mathrm{F} 4$ était purifiée par passage sur Bio-Gel P-roo et une seule activité peptidasique notable était détectée ( $\mathrm{F} 4 a$ ) (tabl. I). Après chromatographie sur Séphadex G-roo seules les fractions $\mathrm{F} 4 a, \mathrm{~F} 3 a$ et $\mathrm{F} 3 b$ présentant une stabilité satisfaisante pendant la conservation à $-30^{\circ} \mathrm{C}$ ont été étudiées; les peptidases ainsi obtenues seront respectivement désignées $\mathrm{AP}$ I, $\mathrm{AP}_{2}$ et $\mathrm{AP}_{3}$ (tabl. I). Cette dernière étape de purification permet d'estimer leur poids moléculaire respectivement à environ too 000, 7x ooo et 69000 daltons.

\section{2. - Caractérisation partielle des aminopeptidases}

Activités en fonction du $p H$.

La peptidase AP I présente un optimum d'activité envers la $\mathrm{LNA}$ de $\mathrm{pH} 5,0$ à 6,0 (fig. 3). Elle est active dans une large zone de $\mathrm{pH}$ puisque les activités mesurées à $\mathrm{pH} 4$, Io ou $\mathrm{pH} 8,2$ représentent encore respectivement 33 , ou $42 \mathrm{p}$. Ioo de cette 

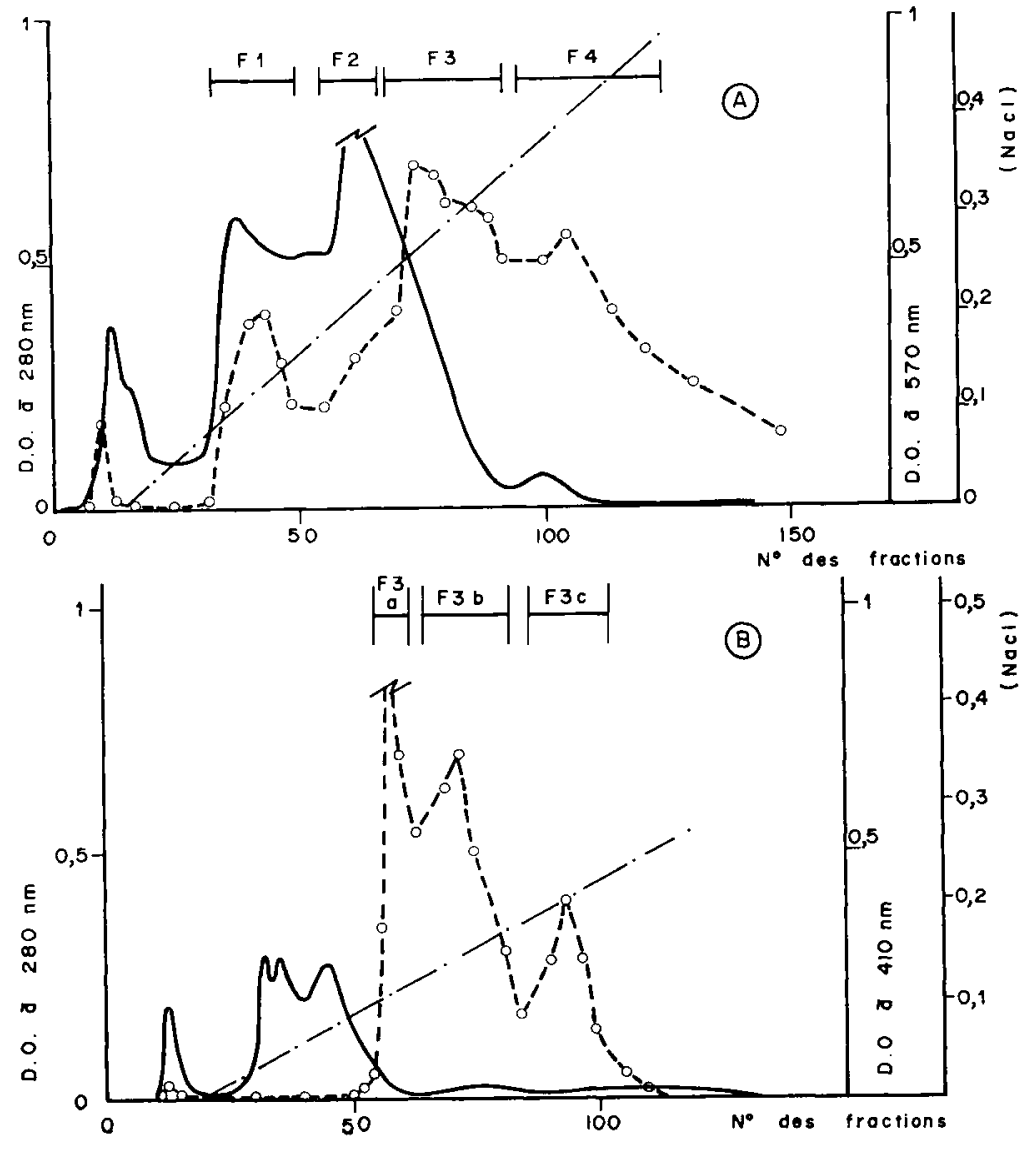

FIG. 2.

A : Chromatographie sur DEA E-cellulose DE-23 de l'extrait peptidasique après Sepharose $6 B$.

Colonne $50 \times 2 \mathrm{~cm}$; volume des fractions $15 \mathrm{ml}$; débit $45 \mathrm{ml} / \mathrm{h}$; tampon Tris-maléate de sodium 0,005 M ; pH 7,10.

$\mathrm{B}$ : Chromatographie sur ECTEOLA-cellulose de l'extrait F 3 précédent.

Colonne $35 \times 2 \mathrm{~cm}$; volume des fractions : $9,7 \mathrm{ml}$; débit $38 \mathrm{ml} / \mathrm{h}$; tampon Tris-maléate de sodium $0,005 \mathrm{M} ; \mathrm{pH} 7, \mathbf{1 0}$.

DO à $280 \mathrm{~nm}=$ élution des protéines ;

o- - - D DO à $570 \mathrm{~nm}$ (substrat : peptides de caséine) = activité peptidasique ;

o-- - O DO à 410 $\mathrm{nm}$ (substrat : LNA) = activité peptidasique ;

$\cdot-\cdot \mathrm{NaCl}=$ pente du gradient de concentration en $\mathrm{NaCl}$. 
activité maximum mesurée à $\mathrm{pH} 5,0$. La vitesse d'hydrolyse de la LNA par la peptidase $\mathrm{AP} 2$ est maximum à $\mathrm{pH} 7,0$ (fig. 3). La vitesse de la réaction mesurée à $\mathrm{pH} 6,0$ ou pH 8,6 représente encore respectivement 54 ou $5^{6} \mathrm{p}$. roo de celle obtenue à $\mathrm{pH} 7,0$. Enfin, la peptidase AP 3 a un $\mathrm{pH}$ optimum d'activité de 8,o. La vitesse d'hydrolyse

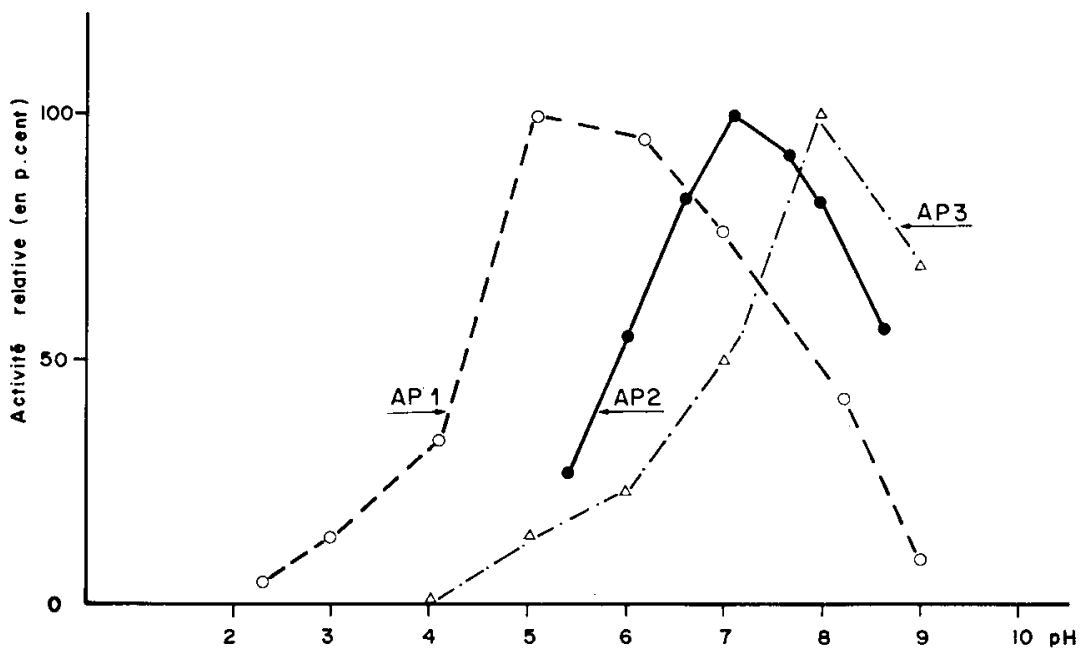

FIG. 3. - Influence du pH sur les différentes activités peptidasiques de $\mathrm{S}$. lactis

Activité mesurée sur la LNA. Les différents pH étaient obtenus dans les tampons o,05 M $\mathrm{KCl}-\mathrm{HCl}(\mathrm{pH} 2,0$ à 3,5$)$, acétate de sodium ( $\mathrm{pH} 4, \mathrm{o}$ à 5,4$)$,

Tris-maléate ( $\mathrm{pH} 5,4$ à 8,5 ), borate de sodium-KCl ( $\mathrm{pH} 8,5$ à 9,0 ).

de la LNA à pH 9,o représente encore $69 \mathrm{p}$. Ioo de cette vitesse maximum. Ces comparaisons ne rendent compte, évidemment, que d'un phénomène global puisque d'une part, il n'a pas été effectué d'études portant ni sur $k_{\text {cat }}$ ni sur $k_{m}$ et que, d'autre part, il faudrait tenir compte aussi de la stabilité de l'enzyme.

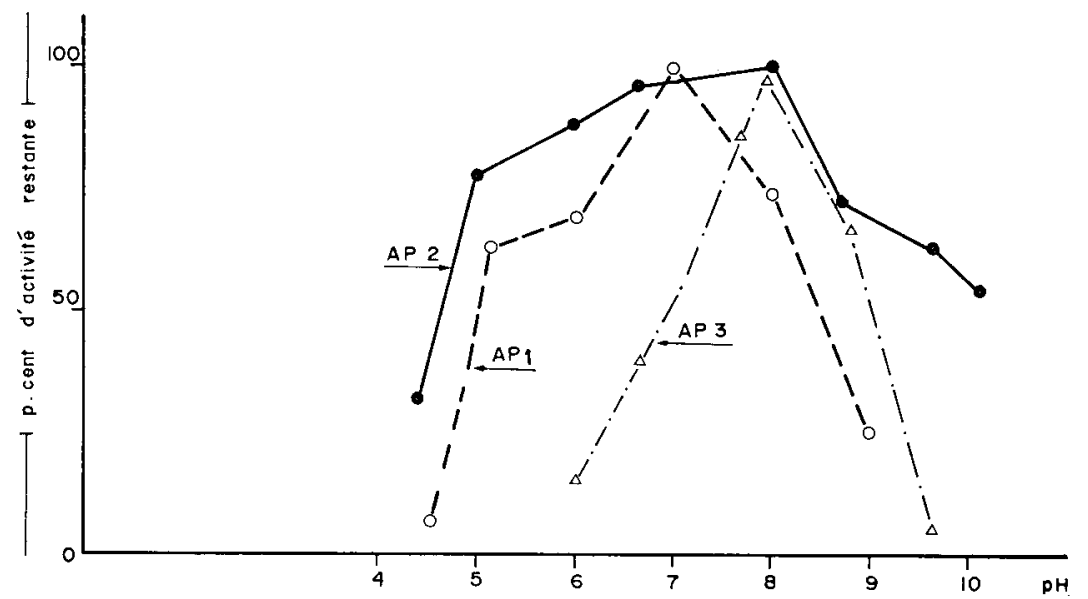

FIG. 4. - Stabilité des aminopeptidases de $\mathrm{S}$. lactis à différents $p H$.

L'activité restante était mesurée après incubation des différentes peptidases pendant $30 \mathrm{mn}$ à $37^{\circ} \mathrm{C}$ dans des tampons o,o5 M : acétate de sodium ( $\mathrm{pH}_{4,0}$ à 5,4 ), Tris-maléate ( $\mathrm{pH} 5,4$ à 8,5), borate de sodium. $\mathrm{KCl}$ (pH 8,5 à Io, I).

L'activité restante était mesurée au pH optimum des différentes enzymes, sur la LNA comme substrat. 
Stabilité aux différents $p H$.

La peptidase $\mathrm{AP}$ I est complètement stable à $\mathrm{pH} 7,0$. Entre $\mathrm{pH} 5,0$ et $\mathrm{pH} 8,0$ il subsiste plus de 50 p. Ioo de l'activité initiale (fig. 4). La peptidase AP 2 est la plus stable des enzymes étudiées puisqu'entre $\mathrm{pH} 5,50$ et 8,20 l'activité restant après incubation est au moins égale à 80 p. Ioo de l'activité initiale (fig. 4) et qu'après traitement à $\mathrm{pH}$ Io, ro il reste encore $53 \mathrm{p}$. Ioo de celle-ci. Enfin, la peptidase $\mathrm{AP} 3$ est peu stable avec un optimum de stabilité à $\mathrm{pH} 8,0$.

\section{Influence de la température.}

L'hydrolyse maximum de la L,NA, par la peptidase AP I, était obtenue à $45^{\circ} \mathrm{C}$ (fig. 5). Les activités mesurées à $50^{\circ} \mathrm{C}$ ou $30^{\circ} \mathrm{C}$ ne représentaient plus respectivement que 87 ou $4^{8} \mathrm{p}$. roo de cette activité maximum. La pente de la droite obtenue dans la représentation graphique de la loi d'Arrhenius (MOELWYN-HughEs, I950) (fig. 5 A)

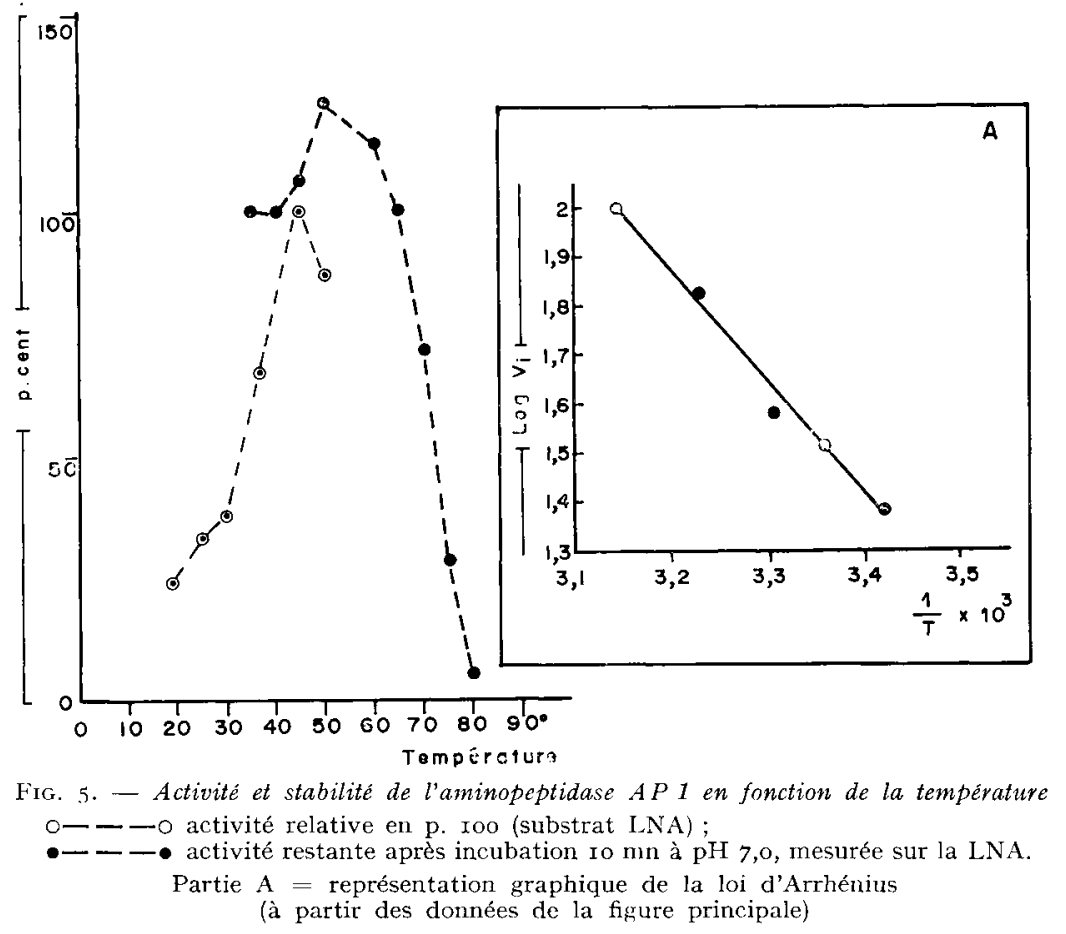

permet de calculer l'énergie apparente d'activation $\mathrm{E}_{\mathrm{A}}$ qui est égale à Io ooo calories/ mole. D'autre part, nous avons déterminé les optima de température des peptidases $\mathrm{AP} 2$ et $\mathrm{AP} 3$ qui sont respectivement, sur la LNA, $45^{\circ} \mathrm{C}$ et $40^{\circ} \mathrm{C}$.

Les expériences d'inactivation thermique ont été réalisées à $\mathrm{pH} 7,0$ pour l'enzyme $\mathrm{AP}$ I et à $\mathrm{pH} 8,0$ pour les peptidases $\mathrm{AP} 2$ et $\mathrm{AP} 3$, en tampon Tris-maléate de sodium $0,05 \mathrm{M}$. L'activité restante était dosée à $37^{\circ} \mathrm{C}$ comme décrit précédemment au $\mathrm{pH}$ optimum d'activité des différentes peptidases, en tampon Tris-maléate de sodium $0,05 \mathrm{M}$.

L'enzyme AP I semble stable jusqu'à $65^{\circ} \mathrm{C}$ après incubation à $\mathrm{pH} 7,0$ pendant 
Io mn. Il faut noter que cette " thermostabilité " peut être due à une réaction d'activation d'une forme zymogène puisqu'un chauffage à $45^{\circ}, 50^{\circ}$ ou $60^{\circ} \mathrm{C}$ permet de retrouver des activités supérieures à l'activité initiale (fig. 5). D'autre part, la peptidase $\mathrm{AP} 3$ semble être, elle aussi relativement thermostable puisque après incubation à $5^{\circ} \mathrm{C}$ aucune activité n'était perdue et qu'après chauffage à $70^{\circ} \mathrm{C}$ pendant $\mathrm{I} 5 \mathrm{mn}$ on retrouvait $50 \mathrm{p}$. Ioo de l'activité initiale.

\section{Influence de certains inhibiteurs.}

Les peptidases isolées de $S$. lactis ont les caractères de métallo-enzymes puisqu'une forte inhibition est obtenue avec 1'o-phénanthroline ou l'EDTA (tabl. 2). Cependant ce caractère n'est vraiment absolu que pour l'enzyme $\mathrm{AP} 3$, la peptidase $\mathrm{AP} 2$ n'étant que peu sensible à $1^{\prime} \mathrm{ED} \mathrm{D}^{\prime} \mathrm{TA}$ même à une concentration de $\mathrm{Io}^{-2} \mathrm{M}$. De plus, la peptidase AP 3 présente une grande sensibilité aux composés réducteurs puisque la cystéine et le $\beta$-mercaptoéthanol l'inhibent complètement (tabl. 2). Les

TABLEAU 2

Effet des inhibiteurs sur les aminopeptidases de $\mathrm{s}$. lactis

\begin{tabular}{|c|c|c|c|}
\hline & $A P 1$ & $\mathrm{AP} 2$ & $\mathrm{AP} 3$ \\
\hline $\mathrm{DFP} \ldots \ldots \ldots \ldots$ & 100 & 100 & 100 \\
\hline Acide iodoacétique $\ldots \ldots$ & $8{ }^{\prime}$ & & \\
\hline$p$-СМВ $\ldots \ldots \ldots \ldots$ & 51,5 & 45,5 & 100 \\
\hline$\beta$-mercaptoéthanol ....... & 100 & 60 & 0 \\
\hline Cystéine $\ldots \ldots \ldots \ldots \ldots$ & & 65,5 & 3,2 \\
\hline EDTA $\ldots \ldots \ldots \ldots \ldots$ & 13 & 45,5 & 0 \\
\hline O-phénanthroline ........ & 13 & 25 & 0 \\
\hline
\end{tabular}

Les enzymes étaient incubées en présence de l'inhibiteur $10^{-2} \mathrm{M}$, à pH 7,10 dans du tampon Tris-maléate de sodium $0,05 \mathrm{M}$ à $\mathrm{pH} 7,10$, à $37^{\circ} \mathrm{C}$ pendant $180 \mathrm{mn}$. L'activité restante était dosée sur la LNA en tampon Tris-maléate.

Les résultats sont exprimés en p. 100 de l'activité initiale.

résidus séryles ne doivent pas intervenir dans le site actif des trois peptidases puisqu'il n'y a pas d'inhibition par le DFP. Nous n'avons obtenu aucune réactivation par les ions $\mathrm{Ca}^{++}, \mathrm{Co}^{++}, \mathrm{Mn}^{++}, \mathrm{Mg}^{++}, \mathrm{Zn}^{++}$ou $\mathrm{Fe}^{++}$, des peptidases inhibées par 1'EDTA. D'autre part, les ions $\mathrm{Zn}^{++}, \mathrm{Mn}^{++}$ou $\mathrm{Mg}^{++}$à une concentration de $\mathrm{IO}^{-3} \mathrm{M}$ entraînent respectivement une inhibition de Ioo, 88,5 ou 42,5 p. Ioo de l'activité de la peptidase $\mathrm{AP}$ 2. Par contre le $\mathrm{p}-\mathrm{CMB}$ inhibe partiellement les enzymes AP I et AP 2, cette dernière étant sensible de plus au $\beta$-mercaptoéthanol et à la cystéine (tabl. 2), ce qui peut être en faveur de résidus sulfhydryles dans le site actif ou de leur présence nécessaire à la conformation active de la protéine enzymatique.

Spécificité du substrat.

Les trois peptidases de $S$. lactis étudiées dans ce travail sont de type aminopeptidasique (EC 3.4.I). En effet, elles hydrolysent soit des peptides de poids molécu- 
laires variés, soit des dérivés d'acides aminés — tels que la leucine-para-nitro-anilide et la phénylalanine amide - à l'extrémité - $\mathrm{NH}_{2}$ libre ; d'autre part, elles sont incapables d'hydrolyser des substrats dont le résidu $\mathrm{NH}_{2}$-terminal est bloqué, tels que les dipeptides de type $\mathrm{Z}-\mathrm{X}-\mathrm{Y}$ ou $\mathrm{Z}-\mathrm{X}^{\prime}-\mathrm{Y}^{\prime}-\mathrm{NH}_{2}$ (tabl. 3).

\section{TABLEAU 3}

Étude comparative de l'activité des peptidases de $\mathrm{S}$. lactis sur différents substrats

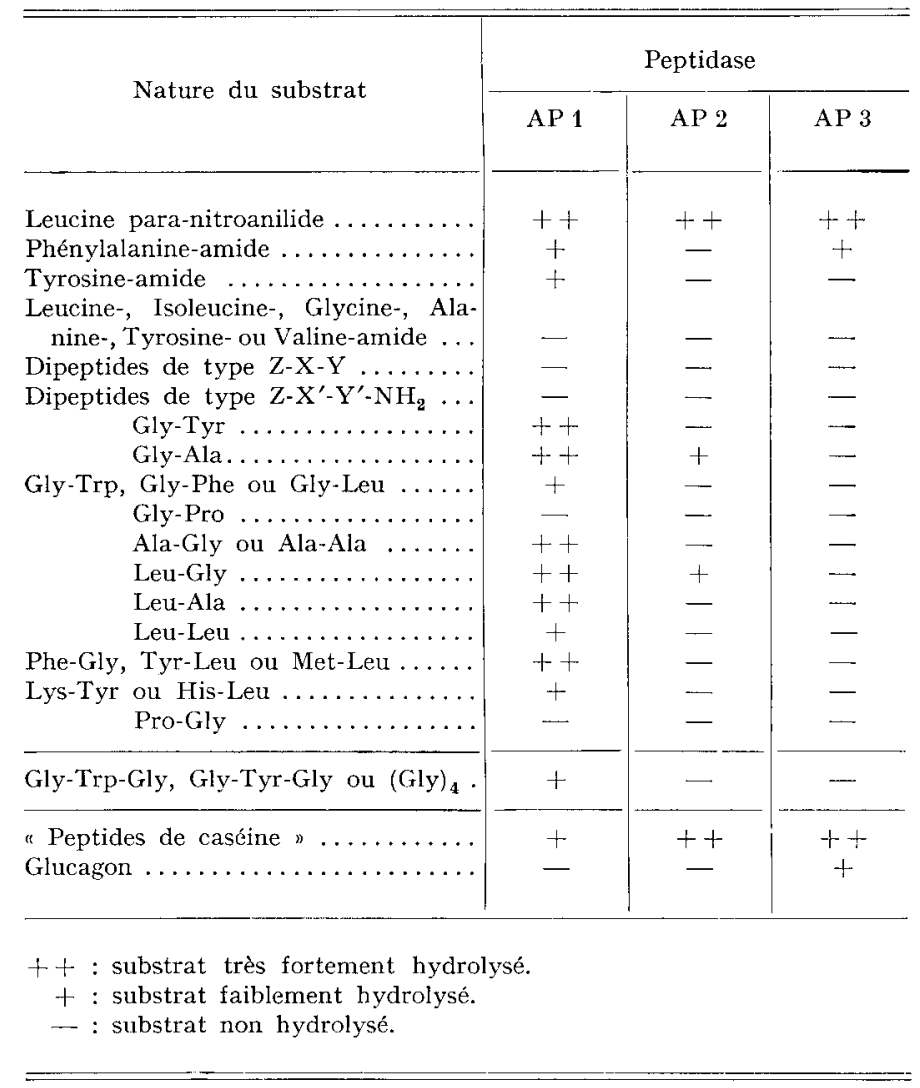

L'aminopeptidase AP I se distingue très nettement des deux autres puisqu'elle hydrolyse tous les dipeptides testés, à l'exclusion cependant de la Pro-Gly ou de la Gly-Pro, alors que la peptidase AP 3 n'en hydrolyse aucun. Par contre, pour ces trois enzymes, les amides des acides aminés ne représentent pas de bons substrats (tabl. 3).

\section{3. - Mise en évidence d'une activité carboxypeptidasique}

L'extrait cellulaire non purifié S I (tabl. I) présente une activité carboxypeptidasique mise en évidence par l'hydrolyse des dipeptides monosubstitués Z-Gly-Letu, Z-Gly-Phe, Z-Gly-Ala et Z-Glu-Tyr. Après chromatographie d'une partie aliquote d'extrait S I sur Bio-gel P-I5o (fig. 6 et tabl. r) une seule activité carboxypeptida- 
sique est éluée, dont le poids moléculaire peut être estimé à 64400 daltons environ. D'autre part, il a été établi qu'à pH 7, Io la vitesse d'hydrolyse du substrat Z-Gly-Leu, par cette carboxypeptidase, est 4 fois supérieure à celle mesurée à $\mathrm{pH} 5,5^{\circ}$.

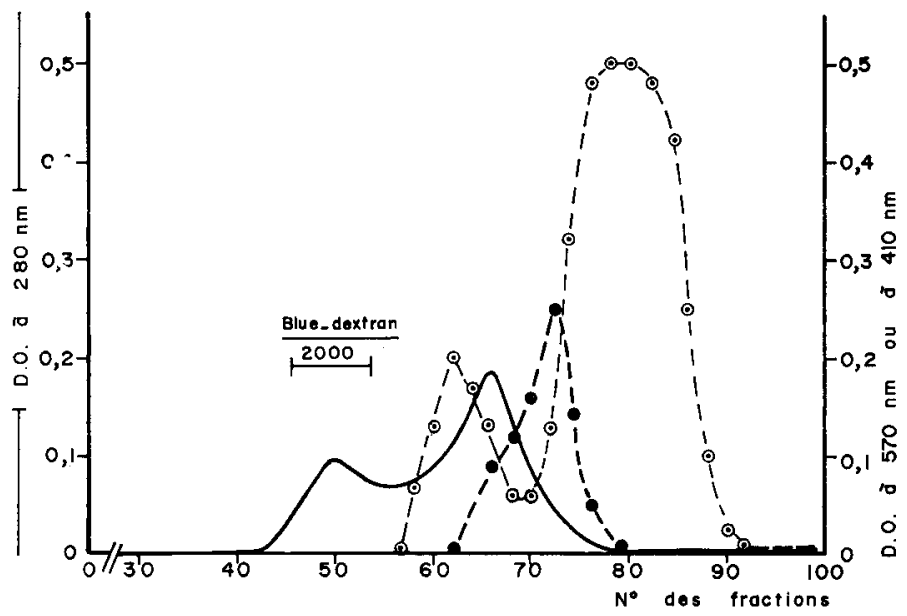

FIG. 6. - Chromatographie sur Bio-Gel P-150 de l'extrait S 1 (cellules traitées aux ultra-sons)

Colonne $88 \times 4 \mathrm{~cm}$; volume des fractions $5,2 \mathrm{ml}$; débit $27 \mathrm{ml} / \mathrm{h}$; tampon Tris-maléate de sodium 0,005 $\mathrm{M} ; \mathrm{pH} 7$, io DO à $280 \mathrm{~nm}=$ élution des protéines;

- - - DO à 4 IO $\mathrm{nm}=$ activité aminopeptidasique (substrat LNA) ;

$\bullet-\longrightarrow$ DO à $570 \mathrm{~nm}=$ activité carboxypeptidasique (substrat Z-Gly-Leu).

\section{DISCUSSION E'T CONCLUSIONS}

Comme les levures de brasserie (FÉLIx et BROUILLET, Ig66) ou les levures de boulangerie (HAYAShI, Aibara et HATA, I970), Saccharomyces lactis possède tune activité carboxypeptidasique liée aux cellules, capable d'hydrolyser des substrats tels que le Z-Gly-Leu, le Z-Gly-Phe ou le Z-Glu-Tyr dans la zone des $\mathrm{pH}$ voisins de la neutralité. Cette carboxypeptidase a un poids moléculaire apparent (64 400 daltons) plus élevé que celui des enzymes du même type isolées des organes animaux qui est par exemple de 34472 daltons pour la carboxypeptidase A (HAR'́suck et LiPSCOMB, 197I) ou de 34300 daltons pour la carboxypeptidase B (Folk, I97I). Par contre, cette propriété la rapproche d'une carboxypeptidase acide isolée d'Aspergillus oryzae qui a un poids moléculaire de 61 ooo daltons (NAKADAI, NASUNo et IGUCHI, I972).

Très peu d'études ont été consacrées aux aminopeptidases des levures, alors que de nombreuses enzymes de ce type ont été déjà purifiées et caractérisées aussi bien à partir de tissus animaux (SMrTh et HII,L, I960) qu'à partir de cellules de différentes espèces bactériennes en particulier Escherichia coli (DICK, MATHEson et WANG, I970 ; VoGT, I970; Yaron et Mlynar, I968), Pseudomonas (Freund, I969, Aeromonas proteolytica (PRESCOTT et al., I97I), Bacillus stearothermophilus (RONCARI et ZUBER, 1969), B. subtilis var. amylosacchariticus (Matsumura et al., I971) ou Streptococcus thermophilus (RABIER et Desmazeaud, I973). Cependant des activités aminopeptidasiques ont été déterminées ou isolées chez S. cerevisiae (BERGER et JoHNSON, I939; 
Smith, I95I ; Sicho et KaS, 1970) ou S. cerevisiae var. ellipsoideus (CoRdonNier, rg66).

Comme S. cerevisiae var. ellipsoideus (CoRdonnIER, I966), S. lactis possède des aminopeptidases dont le $\mathrm{pH}$ optimum est situé près de la neutralité ou légèrement alcalin. Par cette propriété ces enzymes se rapprochent de l'aminopeptidase de $B$. stearothermophilus (RONCARI et ZUBER, I969) qui possède vis-à-vis du substrat LNA un $\mathrm{pH}$ optimum de $7,5^{\circ}$.

Comme de nombreuses aminopeptidases que ce soit la leucine-aminopeptidase du rein de porc ( $\mathrm{EC}$ 3.4.I.I) (SMITH et HILL, I960), ou celles d'origine bactérienne (Rabier et Desmazeaud, I973; Freund, I969; Moser, Roncari et Zuber, I970) ou celle mise en évidence chez $S$. cerevisiae var. ellipsoideus (CoRDONNIER, I966) les peptidases de $S$. lactis sont inhibées par les complexants tels que l'E,DTA ou l'o-phénanthroline et par certains cations tels que $\mathrm{Zn}^{++}$. Ce sont donc vraisemblablement des métallo-enzymes (cependant la présence de métal n'a pas été recherchée, comme i1 serait possible de l'effectuer par adsorption atomique par exemple). De façon semblable à l'aminopeptidase de $S$. cerevisiae, étudiée par GRASSMAN et DYCKERHOFF (I928) (cités par CoRdonNiER, I966), qui était inhibée par le $\mathrm{SH}_{2}$, deux aminopeptidases de $S$. lactis sont inhibées par des composés sulfhydryles tels que le $\beta$-mercaptoéthanol ou la L-cystéine.

L'énergie apparente d'activation $\mathrm{E}_{\mathrm{A}}$ (de la réaction d'activation) de l'aminopeptidase AP I de $S$. lactis qui est égale à Io ooo cal./mole sur le substrat INA est plus élevée que celle obtenue pour l'aminopeptidase de S. thermophilus (RABIER et DeSMAZEAUD, 1973) sur le même substrat. Par contre elle est à rapprocher de celle trouvée chez certaines protéases bactériennes telles que celle de Micrococcus caseolyticus (Desmazeaud et Hermier, I968) ( $\mathrm{E}_{\mathrm{A}}=9$ Ioo cal./mole avec la caséine comme substrat) ou de $S$. faecalis var. liquefaciens (ShuGART et BECK, I964) $\left(\mathrm{E}_{\mathrm{A}}=9060 \mathrm{cal} /\right.$ mole) avec l'hémoglobine comme substrat. Ces deux dernières valeurs ne sont d'ailleurs citées qu'à titre d'illustration, la comparaison n'étant pas possible puisque les études n'ont pas mis en œuvre les mêmes substrats.

Les aminopeptidases de $S$. lactis possèdent des poids moléculaires relativement faibles pour cette catégorie d'enzymes. Cette propriété les rapproche des aminopeptidases de S. thermophilus (Rabifr et Desmazeaud, I973) ou Pseudomonas (FREUND, I969). Il est en effet très inférieur à celui généralement observé pour ce type d'hydrolases. En effet, les autres aminopeptidases ont un poids moléculaire égal ou supérieur à 300 o00 (SMItr et HILL, I960; VOGT, I970; MOSER, RONCARI et ZUBER, I970). Il faut remarquer que ces poids moléculaires élevés traduisent pour les aminopeptidases des formes polymères dont les monomères ont, par contre, un poids moléculaire comparable à celui des aminopeptidases de S. lactis.

Enfin, peu de données existant dans le domaine de la spécificité des aminopeptidases des levures, seule la peptidase $\mathrm{AP} 2$ pourrait être comparée à l'activité déterminée chez S. cerivisiae var. ellipsoideus (CoRdonniER, I966) par le fait qu'elles hydrolysent la Leu-Gly mais non l'Ala-Gly ou la Gly-Leu. En conclusion, les aminopeptidases de $S$. lactis présentent le caractère original de ne pas hydrolyser la plupart des amides d'acides aminés tout en attaquant la leucine-para-nitro-anilide; d'autre part, à l'exception de l'aminopeptidase AP I, les dipeptides ne leur sont pas substrats. 


\title{
REMERCIEMENTS
}

Nous remercions M. J. Hermier pour ses suggestions et ses critiques au cours de la rédaction du manuscrit, ainsi que M. C. Boulllanne qui a eu l'obligeance de réaliser la culture en fermenteur et $M^{1 l e}$ Jeanne Fournaud qui a mis à notre disposition l'appareil de traitement aux ultrasons.

\section{SUMMARY}

\section{REVEIATION AND PARTIAL CHARACTERIZATION OF VARIOUS PEPTIDASES IN SACCHAROMYCES LACTIS}

\begin{abstract}
After cells were ground supersonically, carboxypeptidase-type activity (EC 3.4 .2$)$ was revealed at $7.0 \mathrm{pH}$ on synthetic substrates Z-Gly-Leu, Z-Gly-Phe and Z-Glu-Tyr. After elimination of nucleic acids and precipitation of ammonium sulphate, three aminopeptidase-type activities (EC 3.4.I) were revealed on the Leucine-para-nitro-anilide substrate (LNA) and isolated by DEAE-cellulose and ECTEOLA-cellulose chromatography. Aminopeptidases (AP I, AP 2, AP 3) have the characters of metalloenzymes: EDTA and $O$-phenanthroline strongly inhibit AP I and AP 2 peptidases, and completely inhibit AP 3 enzyme. The latter is also inactivated by cysteine and $\beta$-mercaptoethanol. AP I, AP 2 and $\mathrm{AP}_{3}$ peptidases have an optimum $\mathrm{pH}$, respectively, of : 5.0, 7.I and 8.0 and present a maximum stability of 7.0 to $8.0 \mathrm{pH}$. Their optimal temperature of LNA hydrolysis is 40 to $45^{\circ} \mathrm{C}$ (apparent activation energy of AP I peptidase is 10 ooo cal $/ \mathrm{mole}$ ). AP 3 enzyme is stable at $50^{\circ} \mathrm{C} ; 5^{\circ} \mathrm{p}$. Ioo of the activity is destroyed after incubation at $70^{\circ} \mathrm{C}$ for I $5 \mathrm{~min}$. AP I peptidase is stable up to $65^{\circ} \mathrm{C} ; 28 \mathrm{p}$. roo of the activity is destroyed after incubation at $70^{\circ} \mathrm{C}$ for $10 \mathrm{~min}$. These peptidases also have different specificity in regard to the hydrolysis of many synthetic peptides.
\end{abstract}

\section{RÉFÉRENCES BIBLIOGRAPHIQUES}

Berger J., Johnson M.-J., I939. Metal activation of peptidases. J. Biol. Chem., 130, 64 I-654.

Cordonnier R., I966. Recherches sur l'amino-carboxypeptidase (dipeptidase) de Saccharomyces cerevisiae var. ellipsoideus. Ann. Technol. agr., 15, H. S. I, I-I I4.

DesmazeAuD M.-J., r972. Contribution à l'étude de la spécificité de la papaīne : hydrolyse du glucagon. Biochimie, 54, I IO9-III4.

Desmazeaud M., Hermier J., rg68. Isolement, purification et propriétés d'une protéase exocellulaire de Micrococcus caseolyticus. Ann. Biol. anim. Bioch. Biophys., 8, 565-577.

Desmazeaud M.-J., Hermier J.-H., r972. Isolement et détermination de la composition qualitative de peptides issus de la caséine, stimulant la croissance de Streptococcus thermophilus, Eur. J. Biochem., 28, $190-198$.

Devoyod J.-J., Desmazeaud M., r970. Les associations microbiennes dans le fromage de Roquefort. I. Action des entérocoques vis-à-vis des streptocoques lactiques et des Leuconostoc. Nature des substances stimulantes produites par Streptococcus faecalis var, liquefaciens. Le lait, 50, 374-39o.

Devoyod J.-J., Desmazeaud M., I97I. Les associations microbiennes dans le fromage de Roquefort. III. Action des entérocoques et des levures fermentant le lactose vis-à-vis des lactobacilles. Le Lait, 51, $399-4$ I 5 .

Devoyod J.-J., Sponem D., I970. La flore microbienne du fromage de Roquefort. VI. Les levures. Le Lait, 50, 524-543.

Dick A. J., Mathejon A. T., WANG J. H., I970. A ribos mal-bound aminopeptidase in Escherichia ccli B : purification and proparties. Can. J. Biochem., 48, I I8I-II88.

Ferix F., Brouillet N., ig55. Purification et propriétés de deux peptidases de levure de brasserie. Biochim. Biophys. Acta, 122, r27-r44.

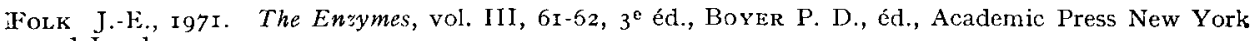
and Londor. 
Freund T. S., I969. The proteolytic enzymes of a new marine Pseudomonas. In a dissertation (for the degree of Doctor of Philosophy in Chemistry), Lehigh University, U. S. A.

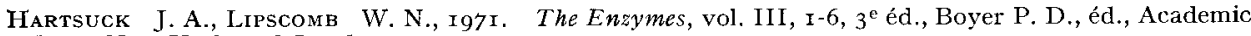
Press New York and London.

Hata T., Hayashi R., Dor E., i 967 . Purification of yeast proteinases. Part. I. Fractionation and some properties of the proteinases. Agr. Biol. Chem., 31, I50-I 59.

Hayashi R., Aibara S., Hata T., I97o. A unique carboxypeptidase activity of yeast proteinase C. Biochim. Biophys. Acta, 212, 359-36r.

Kamada M., Oda K., Murao S., I972. The purification of the extracellular acid protease of Rhodotorula glutinis K-24 and its general properties. Agr. Biol. Chem., 36, ro95-1 Io2.

Lenney J. F., Dalbec J. M., I967. Purification and properties of two proteinases from Saccharomyces cerevisiae. Arch. Biochem. Biophys., 120, 42-48.

Maddox I. S., Hough J. S., I970. Proteolytic enzymes of Saccharomyces carlsbergensis. Biochem. J., 117, $843-852$.

Matsumura Y., Minamiura N., Fumumoto J., Yamamoto T., I97I. Intracellular peptidase of Bacillus subtilis. III. Effects of metal ions on activity and specificity of aminopeptidase of Bacillus subtilis. Agr. Biol. Chem., 35, 975-982.

Monlwyn-Hughes E. A., 1950. The Enzymes, vol. 1, 28-78, Ire éd., Sumner J. B. et Myrbach K., éd., Academic Press, New York.

Moore S., Stern W. H., I954. A modified ninhydrin reagent for the photometric determination of amino acids and related compounds. J. Biol. Chem., 211, g07-913.

Moser P., Roncari G., Zuber H., I97o. Thermophilic aminopeptidases from B. stearothermophilus. II. Aminopeptidase-I (API) $=$ physicochemical properties; thermostability and activation ; formation of the apoenzyme and subunits. Int. J. Protein Res., 2, I9I-207.

Nakadai T., Nasuno S., Iguchi N., I972. Purification and properties of acid carboxypeptidase III from Aspergillus oryzae. A gr. Biol. Chem., 36, 1481-I488.

Prescott J. M., Wilkes S. H., Wagner F. W., Wilson K. J., I97i. Aeromonas aminopeptidase. Improved isolation and some physical properties. J. Biol. Chem., 246, I 756-1764.

Rabier D., Desmazeaud M. J., I973. Inventaire des différentes activités peptidasiques intracellulaires de Streptococcus thermophilus. Purification et propriétés d'une dipeptide-hydrolase et d'une aminopeptidase. Biochimie, 55, 389-404.

Roncari G., Zuber H., I969. Thermophilic aminopeptidases from Bacillus stearothermophilus. I. Isolation, specificity and general properties of the thermostable aminopeptidase. I. Int. J. Protein Res., $1,45-6 \mathrm{r}$.

Shugart L. R., Beck R. W. Purification and activity of proteinase of Streptococcus faecalis var. liquefaciens. J. Bacteriol., 88, 586-590.

Sicho V., KAS J., I970. Leucine aminopeptidase activity in dying animal and plant tissue, and in yeast cells. Vys. Skol. Chem. technol. Praze, E 29, 8-22.

Smith E. L., r95I. The Enzymes, vol. I, 793-872, Ire éd., Sumner J. B. et Myrbach K., éd., Academic Press, New York.

Sмith E. L., Hill R. L., I960. The Enzymes, vol. 4 A, 37-62, Boyer P. D., Lardy H. et Myrbach K., éd., Academic Press, New York and London.

Vogt V. M., 197o. Purification and properties of an aminopeptidase from Escherichia coli. J. Biol. Chem., 245, 4760-4769.

YARON A., MlYNAR D., I968. Aminopeptidase-P. Biochem. Biophys. Res. Comm., 32, 658-663. 